\title{
Dipeptidyl-Peptidase 4 (Cd26): a Possible Therapeutic Target in Covid-19
}

\author{
Galimberti $\mathrm{S}^{1 *}$, Morabito $\mathrm{F}^{2,3}$, Gentile $\mathrm{M}^{4}$, Baratè $\mathrm{C}^{5}$, Benedetti $\mathrm{E}^{5}$, Buda $\mathrm{G}^{\mathbf{1}}$ and Petrini $\mathrm{M}^{\mathbf{1}}$ \\ ${ }^{1}$ Department of Clinical and Experimental Medicine, University of Pisa, Italy \\ ${ }^{2}$ Biothecnology Research Unit, AO of Cosenza, Italy
}

${ }^{3}$ Hematology and Bone Marrow Transplant Unit, Hemato-Oncology Department, Israel

${ }^{4}$ Hematology Unit, AO of Cosenza, Italy

${ }^{5}$ UO Hematology, AOUP, Italy

*Corresponding author: Sara Galimberti, Department of Clinical and Experimental Medicine, UO Hematology, University of Pisa, Pisa, Italy

\begin{abstract}
SARS-COV-2, a novel $\beta$-coronavirus, is the cause of a severe inflammatory infectious disease of the respiratory tract (COVID-19). The spread has already taken on pandemic proportions, affecting over 2,5 million people and causing more than 170,000 deaths. The mechanisms and strategies underlying the virus power of penetrating human cells and causing the well-known spectrum of diseases induced by SARS-COV-2 have been explored worldwide. Two host receptors able to specifically inducing virus-host linkage, entry and, consequently, productive infection, have been suggested to interact with the outer membrane spike viral glycoprotein: the angiotensin converting enzyme 2 (ACE2) and the dipeptidyl-peptidase 4 (DPP4), also known as CD26. Both these receptors are highly expressed on several human tissues (i.e. kidney, pancreas, gut, lung, endothelium, pleura, myocardium, connective tissue) accounting for the variable clinical manifestations of COVID-19. CD26 is also over-expressed in stimulated T, B, and NK cells, thus representing an activation marker of the immune system. However, CD26 is not only the functional host receptor for SARSCoV-2. Indeed, published data available from the previous SARS-CoV and MERS-CoV outbreaks showed that CD26 is also utilized for sustaining inflammation and counteracting the host immune response. Specifically, through CD26, coronavirus may increase inflammatory cytokine production, down- modulate the autophagy, and increase levels of adenosine, hence further deactivating the host immune response. Thus, compounds able to inhibit the DPP4/CD26 pathway might be useful against COVID-19. In this respect, promising therapeutic approaches could include: 1) DPP4 inhibitors, such as sitagliptin, already used for treating diabetic patients; 2) Begelomab, the anti-CD26 monoclonal antibody already successfully employed in the treatment of graft-versus-host disease, and 3) adenosine deaminase agonists, already used in the immunodeficiencies sustained by the adenosine deaminase gene mutations. The article will review some pathogenic landscapes and will hypothesize some promising drugs to face the COVID-19 emergency.
\end{abstract}

Keywords: COVID-19; SARS, CD26; DPP4; Autophagy; Inflammation; Sitagliptin; Begelomab; Adenosine deaminase

\section{Introduction}

SARS-CoV-2 is a novel $\boldsymbol{\beta}$-coronavirus identified as responsible of the pandemic that from 30 December 2019 to the first 4 months of 2020 caused worldwide more than 170,000 deaths $(45,000$ in USA, 107,000 in Europe and 4,600 in China). This $\boldsymbol{\beta}$-coronavirus shares $80 \%$ of sequence identity with SARS-CoV, responsible for the Asian outbreak in 2002, and with MERS-CoV, that sustained the Middle East outbreak in 2012 [1]. Starting from this similarity, many researchers tempted to adapt the SARS and MERS models to the new severe inflammatory infectious disease of the respiratory tract sustained by SARS-CoV-2 (COVID-19). Nowadays, two host human receptors have been detected as the cause of the virus-host link and the consequent entry into human cells: i) the angiotensin converting enzyme 2 (ACE2) [2], and ii) the dipeptidyl-peptidase 4 (DPP4), also known as CD26 [3]. Both receptors are strongly 
expressed in kidney, heart, pancreas, small intestine, endothelium and lung [4]. In addition, CD26 is recognized as a marker of activated T-B-and NK-cells; moreover, it has been demonstrated to play a further important role in sustaining the hyper-inflammatory status that characterizes COVID-19 [5]. Although the mechanisms allowing the virus entry and replication are not still fully clarified, the endolysosomal network, comprising both clathrin-mediated and clathrin-independent endocytic pathways, might be a key machinery to be considered as a good antiviral therapeutic target [6]. Chlorpromazine, a distinctive clathrin-dependent endocytosis inhibitor with a suppressive effect on cellular entry of both SARS$\mathrm{CoV}$ and MERS-CoV, could be a promising therapeutic option also for COVID-19, further demonstrating that endocytosis is a relevant process for the Coronavirus replication after the proteolytic cleavage of the surface spike (S) protein [7].

Chloroquine, well-known anti-malarial drug, modifying the endosomal-lysosomal $\mathrm{pH}$, seems to prevent SARS-CoV2 replication [8]. Furthermore, teicoplanin, an antibiotic with proved efficacy against MERS-CoV and SARS- CoV via cathepsin pathway inhibition, has been reported as a promising therapeutic strategy for the present outbreak [9]. Typical antiviral drugs have been also used as reasonable therapeutic strategies for COVID-19 patients to block, after endocytic phase, virus replication. In a first report, $76 \%$ of 99 Chinese COVID-19 patients received at least 3 days of antiviral treatment, including oseltamivir $(75 \mathrm{mg}$ every $12 \mathrm{~h}$, orally), ganciclovir $(0.25 \mathrm{~g}$ every $12 \mathrm{~h}$, intravenously), and lopinavirritonavir (500mg twice daily, orally). Although the study seems underpowered, the mortality rate of $11 \%$ leaves still open the debate about the role of these class of drugs in the pandemic [10]. A further therapeutic approach involves biological drugs able to switch off the cytokine storm responsible for the most severe COVID-19 symptoms and clinical signs (capillary leak, respiratory distress, super-infections, myocardial damage), either alone or in combination with low-weight heparins, taken into consideration to limit the pro-thrombotic status characterizing the disease. However, while disseminated intravascular coagulation is quite common in COVID-19 patients, the anti-thrombotic treatment strategy still present weakness to be convincingly introduced in a COVID-19 therapeutic algorithm [11]. COVID-19 patients frequently show lymphopenia, atrophy of spleen and, more importantly, elevated levels of proinflammatory cytokines. The huge number of macrophages infiltrating the lung of COVID-19 patients is responsible of the so-called primary cytokine storm, whilst activated $\mathrm{T}$ lymphocytes seem to trigger a subsequent phase [12]. Higher concentrations of naive T helper and Th17 cells, accompanied by a more pronounced reduction of CD8+, NK and Tregs lymphocyte subpopulations have been documented in more severe cases [13]. Moreover, chemokines and adhesion molecules seem to have a fundamental role during SARS-COV-2 infection. In particular, the increased expression of neutrophil and monocyte adhesion molecules, such as LFA-1, Mac-1, VLA-4 and ICAM-1, may contribute to their greater adhesiveness to endothelium, inducing vasoconstriction and thrombotic events, which ultimately may be responsible of the mechanisms underlying cardiotoxicity observed during Coronavirus infection [14].

Chloroquine, largely employed for treating autoimmune diseases [15], was first proposed by rheumatologists for the treatment of COVID-19 patients with the aim to block SARS- CoV-2 endocytic host cell entry via human ACE2 receptor [8]. Tocilizumab, an anti-IL6 antibody, already used in rheumatoid arthritis (AR) [16], Anakinra, an anti-IL1 antibody already effective in the macrophage activation syndrome [17], and anti-TNF alpha antibodies, such as Adalimumab, useful for the treatment of psoriasis [18] are some examples of drugs utilized as compassionate use and published, in some cases as uncontrolled non peer-reviewed studies, with consequent significant limitations and biases. In April 2020, the Italian Drug Agency (AIFA) authorized the compassionate use of Ruxolitinib, a JAK1/2 inhibitor already successfully employed by hematologists for treating myelofibrosis, polycythemia vera and acute graft versus host disease (aGVHD) [19].

The T-lymphocyte dysregulation occurring in aGVHD is similar to that induced by SARS-CoV-2 infection. In particular, $\mathrm{T}$ helper and $\mathrm{T}$ cytotoxic lymphocytes abnormalities (i.e. higher percentage of "naive" and "switched off" cells, higher Th1/Th2 ratio, and reduced level of NK and Tregs cells) [20], are shared by the two diseases. Ruxolitinib, by inhibiting Th1 lymphocytes and reducing secretion of pro-inflammatory cytokines, offered $55 \%$ of responses to steroid-refractory GVHD patients [21]. Based on the assumption that immuno-based COVID-19 injury resembles aGVHD, both from the pathogenetic and the clinical point of view, Ruxolitinib could be tailored in this hard-to-treat setting. In this complex scenario, an actor able to play different leading roles and to make a "strict pact with devil" (the Coronavirus) is the dipeptidyl peptidase 4 (DPP4 or CD26) a $110 \mathrm{KDa}$ protein, whose codifying gene is located on the long arm of chromosome 2 (2q24.3) [22]. his protein, known both in the transmembranal and in the shedding form, might be considered the SARS-COV-2 henchman for several reasons. Indeed, CD26 is not only one of the virus receptor on human epithelial cells, but it also represents an important interface between inflammation, metabolism and host immune response that favors the virusinduced unwanted actions [5], similar to those already documented in autoimmune diseases, cancer, diabetes, atherosclerosis, obesity and cystic fibrosis. Thus, DPP4/CD26 might be a reasonable therapeutic target in the 2020 pandemic, and DPP4 inhibitors, previously utilized in autoimmune settings [23,24], could also be implemented in the COVID-19 therapeutic armamentarium. The aim of this paper was to reviewing the biological bases supporting the hypothesis of an unfavorable strict link between SARS-CoV-2 and DPP4/CD26, and discussing the resulting therapeutic options of avoiding the DPP4/CD26/SARS-CoV-2 cross-talk. 


\section{DPP4/CD26 as Receptor for SARS-Cov-2}

DPP4/CD26 is a glycoprotein belonging to the prolyl oligopeptidase family, able to remove the $\mathrm{N}$-terminal dipeptide from proteins containing proline, glycine or alanine [25]. Due to its enzymatic activity, DPP4/CD26 interacts with many proteins, such as adenosine deaminase, fibronectin, collagen, the chemokine receptor CXCR4, and the tyrosine phosphatase CD45 [26]. CD26 is constitutively expressed on a variety of cell types and tissues, such as prostate, kidney, liver, epithelial cells, and exocrine glands [27]. Thus, some symptoms and signs associated with COVID-19, i.e. nausea, vomit, diarrhea, pneumonia and insulin-resistance, could be explained by the widespread distribution of CD26 as well as ACE2 receptors. CD26 is also expressed on myocardial venules/ capillaries and on pneumocytes, especially in subjects with a history of smoking or a previous chronic lung disease or cystic fibrosis [28]. DPP4/CD26 has been previously reported to be one of the receptors of MERS-CoV, the virus responsible for the outbreak that occurred in the Middle East in 2012. The observation that CD26 is rarely detected in the nasal cavity while more frequently found in distal airways and that the most frequent clinical manifestations during outbreak of 2012 interested the lower respiratory tract, seems to support the relevant role of DPP4 in infections sustained by Coronaviruses [29].

Moreover, CD26 is found mainly on type I and type II lung cells and alveolar macrophages, as well as in the endothelium and pleural mesothelium. Notably, the high CD26 expression seems to be associated with the damage suffered by respiratory tract/lung. In keeping with this observation is the epidemiology of COVID-19. In Italy, $13.7 \%$ of deaths for COVID-19 presented the Chronic Obstructive Pulmonary Disease (COPD) as principal comorbidity. Moreover, $73.8 \%$ and $33.9 \%$ of hospitalized patients suffered by hypertension and diabetes, respectively. Notably, both conditions are characterized by a hyper-inflammatory status potentially inducing DPP4 over-expression. Indeed, during lung chronic diseases, such as COPD and cystic fibrosis, an increased detection of DPP4/CD26 protein in alveolar epithelial cells has been documented [30], suggesting that a higher density of the virus receptor, especially in lung, might be responsible for a more rapid virus entry into the human cells and a consequent more rapid and uncontrollable virus dissemination. This hypothesis is in line with that observed after HIV-1 infection, when the excessive truncation of CCL5 chemokine, exerted by the CD26-positive T lymphocytes, was able to transform CCL5 into a potent inhibitor of the monocyte chemotaxis, thus allowing HIV-1 to replicate easier [31]. Moreover, the strict correlation between CD26 cellular surface density and the viral load has been clearly shown in the MERS-CoV model, where the higher infection susceptibility well correlated with higher CD26 density on the host cells [32]. Moreover, the infection efficiency of the Huh-7 cell line with MERS-CoV was significantly reduced by the cells pre- incubation with the anti-DPP4/CD26 antiserum, but not by the control or the anti-ACE2 serum [33]. Recently, the tridimensional structure of the SARS-CoV-2 S1 glycoprotein allowed to appreciate that SARS-COV-2 specific ligand binds CD26 at the same residues 159 used by SARS-CoV and MERS-CoV (K267, T288, A289, A291, L294, I295, R317, Y322 and D542), but with additional few residues (Q286, I287, N338, V341, R336), so indisputably confirming that CD26 is a fundamental receptor also for the SARS-CoV-2 162 [34]. Two epidemiological observations catalyzed the scientific community interest for the DPP4/CD26. The first deals with the 2002 SARS outbreak. Some South Korean cases were infected by SARS-CoV strain carrying a mutated $\mathrm{S}$ protein, thus impairing the virus binding to human CD26 receptor. Interestingly, the disease appeared less aggressive than in the remaining Korean population infected by the "wild-type" virus [35]. The second consideration, applicable also to the actual pandemic, is that not all individuals living in the same community are equally infected. A possible explanation of this phenomenon could rely on genetically-driven differences, mainly receptors' polymorphisms [36]. However, a recent Chinese publication has disavowed such a consideration, showing neither a clear association between the ACE2 polymorphisms with the different prevalence of COVID-19 infection between male and females, nor that between SARS-CoV-2 cases with mild and severe disease [37]. No data have been produced on DPP4 SNPs yet. Nevertheless, in diabetic patients, a strict correlation between the rs4664443 G>A SNP and plasma levels of CD26 has been reported [38]. Thus, receptor polymorphisms could be worth of consideration also in COVID-19.

\section{DPP4/CD26 as First-Line Player in Inflammation}

Due to its ubiquitous localization [39], DPP4/CD26 might play an important role in the cross-talk between innate and adaptive immunity, as well as between the immune system and specific tissue functions. Indeed, while DPP4 activates lymphocytes, the protein fraction expressed on macrophages binds to adenosine deaminase (ADA), increasing the level of its substrate adenosine, ultimately impairing the host immune response [40]. In the kidney, DPP4/CD26, expressed on T- and B-lymphocytes, macrophages and dendritic cells, has been identified as a costimulatory molecule amplifying the activation of T-cells by cross-linking to its ligand caveolin-1. In DPP4-mutated models, where CD26 enzymatic activity is reduced, no increase of IL2 production (that occurs after T-cell activation) has been detected [41]. The hypothesis of a pathogenetic important cross-talking between inflammation and DPP4/CD26 is also supported by its high expression in several inflammatory diseases, such as cystic fibrosis and COPD, where it sustains the inflammatory milieu [42].

In an animal model of cystic fibrosis, Pseudomonas aeruginosa infection increased the acinar airspace, reduced the septal thickness and caused a significant interstitial edema. Notably, this phenomenon was significantly less appreciated in CD26- 
knockdown compared with CD26-expressing rats [43]. Moreover, DPP4 has been reported to play an important pathogenetic role in autoimmune diseases, such as rheumatoid arthritis (RA) and systemic erythematosus lupus (SLE) [44]. It has been hypothesized that DPP4/CD26 might be the target of some important proteins, such as collagen and fibronectin, that behave like autoantigens. In line with this hypothesis, high levels of anti-CD26 antibodies have been found in patients with RA compared with healthy donors [45]. Interestingly, T-lymphocytes located in the synovial cavities express high levels of CD26 and sustain the production of inflammatory chemokines and cytokines [46]. In patients with allergic asthma, CD26 levels have been found to be elevated, suggesting a role of this peptidase also in the pathogenesis of this allergic disease [47]. Finally, in multiple sclerosis, CD26-positive effector T-cells are significantly increased in all disease subtypes compared with healthy controls, well correlating with the disease aggressiveness [48]. In addition to autoimmune diseases, DPP4/CD26 has been widely studied in diabetes mellitus, where this glycoprotein seems to be implicated in the glucose homeostasis by participating to the catalytic degradation of glucagon-like peptide-1 [49]. DPP4 inhibitors increased the long list of the therapeutic armamentarium against diabetes from 2006. DPP4 inhibitors lower blood sugar levels by inhibiting the degradation of the glucagon-like peptide- 1 and - 2 and of glucose-dependent insulinotropic peptide, and by increasing the insulin secretion [50]. Interestingly, it has been reported a high prevalence rate of COVID- 19 in hospitalized diabetic patients (31.8\%) compared with less severe SARS-COV-2 non- hospitalized cases (5.4\%), thus highlighting a strict correlation between severity of COVID- 19 and this pathological condition [51]. Cancer represents another disease where the inflammation plays an unquestionable role and where CD26 might be a relevant mechanistic function. The relationship between neoplasms and germ-induced inflammation is well recognized. The association between gastric cancer with Helicobacter Pylori infection, cervical cancer with Papillomavirus, nasopharyngeal carcinoma and lymphomas with Epstein-Barr virus are well-known examples. Moreover, pro-inflammatory factors, such as asbestos, nanomaterials, hydrocarbons have been recognized as carcinogenic [52].

DPP4/CD26 has been reported to be overexpressed in prostate cancer [53] and it is used as biomarker for differentiating malignant from benign thyroid disorders [54]. In hematology, high levels of DPP4 have been reported in T-cell acute leukemias [55] and in T-cell lymphomas, where CD26 positivity is considered an indicator of a more aggressive disease phenotype [56]. In chronic myeloid leukemia (CML), CD26 has been recognized as the specific marker of the CD34+/CD38-/CD90+ leukemic stem cell [57]. Our group demonstrated that CD26 is co- expressed with the aberrant BCRABL1 fusion tyrosine kinase and with BMI, a gene belonging to the Polycomb family, involved in the cancer epigenetic control [58]. Noteworthy, CD26-positive CML leukemic stem cells circulate even when patients are in deep molecular response and treatment-free, and they are considered operationally cured [59]. In bone marrow, DPP4, degrading CXCL12, is able to mobilize immature cells from the niche to the peripheral blood [60]. Vildagliptin, a DPP4 inhibitor, reduced the leukemic stem cell mobilization and tumor proliferation in vitro, especially when combined with Nilotinib, a tyrosine kinase inhibitor used as first or subsequent lines of CML treatment [61]. Finally, CD26 expression on neoplastic B-cells in chronic lymphocytic leukemia (CLL) has been considered a marker of disease aggressiveness, correlating with disease clinical stage, b2-microglobulin, LDH and absolute lymphocyte count [62]. Our group confirmed the above-mentioned association with other prognostic biomarkers (i.e. CD38 and ZAP-70 expression, and with IgVH unmutated status), Moreover, a higher CD26 expression $(>10 \%)$ on CLL B-cells predicted a shorter therapy need, while this peptidase was absent or barely detectable in various other peripheral B-cell lymphoid tumours [63].

\section{SARS-Covs, DPP4/CD26 and Autophagy}

As virus replication depends on energy and metabolism of host cells, many researchers focused their efforts to clarify, and possibly identify, metabolic processes used by the SARS-CoV-2 to replicate. The metabolomic profile analysis of monkey epithelial cells after infection by old Coronaviruses showed a significant alteration of 25 metabolites, including glutathione, pyrimidines, and metabolites of aminoacyl-tRNA biosynthesis. The authors of this complex study also observed a low AMP/ATP ratio that reduced the AMP-activated protein kinase levels, with subsequent reduced autophagy as output of the viral infection [64]. Autophagy is a process that, controlling the disposal of damaged organelles, plays important roles in the cellular homeostasis, development, aging and defense from infections. Indeed, bacteria and viruses are sequestered into autophagosomes and then delivered to the lysosomes where they are destroyed. Indeed, once activated by the pro- inflammatory cytokines, autophagy contributes to eliminate infective agents by sustaining the MHC-restricted antigen presentation and by negatively regulating the IL1-dependent inflammatory pathway [65]. Alterations in the autophagy have been reported in chronic inflammatory diseases, such as SLE and Crohn's disease, but also in conditions characterized by increased lung fibrosis, such as the pulmonary hypertension, COPD, and cystic fibrosis [66]. Unfortunately, SARS-CoV-2 as well as other bacteria and viruses can subvert this process to their own benefit [67].

Indeed, the Coronaviruses encode eight accessory proteins (ORFs) that are responsible for different phases of infection. Specifically, ORF-3a and ORF-8a trigger apoptosis, ORF-7a activates NF-кB, ORF3b upregulates the expression of cytokines and chemokines involved in the inflammation, ORF-6 reduces interferon production, ORF-8b induces cellular DNA synthesis, and ORF- $9 \mathrm{~b}$ is involved in the ATG-5-dependent autophagy [68]. The overall final result is the freezing of the host immune response and increased viral replication. It has been reported that old Coronaviruses 
were able to reduce autophagy by using their E3-ligase S-phase kinase-associated 2 (SKP2) protein for blocking the activity of the autophagy-initiating protein Beclin-1 [69], so bypassing the first host defense modality represented by the autophagic process. Recently, autophagy has been reported to be relevant also during the SARS-CoV-2 infection. Similarly to the old Coronavirus, the new one decreases glycolysis and downregulates autophagy by degrading Beclin-1 protein. Notably, spermidine, an autophagy inducer, significantly reduced the SARS-CoV-2 replication in a vitro model [70]; this finding represents a proof of concept on the role exerted by autophagy during virus infection.

The autophagy might be considered another meeting point between Coronavirus and DPP4/CD26. Indeed, vildagliptin, a DPP4 inhibitor, blocking the Beclin-1/Bcl-2 interaction and increasing the levels of the pro-autophagy LC3 protein, significantly prolonged survival of mice by restoring the autophagic response in the infarcted myocardium [71]. Analogously, in a murine model of limb ischemia, sitagliptin, another DPP4 inhibitor, restored autophagy in endothelial cells, improving angiogenesis and the consequent limb blood perfusion [72]. Overall considered, these data support the concept that CD26 receptor is used as a 'slave' by SARS-CoV-2, exploiting this pathway to induce hyper-inflammation and to reduce autophagy, thus cutting out two physiological routes of host defending against the virus, with the negative consequence of immune response impairment and the ultimate tissue damage.

\section{OUTLOOK: the Employ of DPP4/CD26 Inhibitors Against COVID-19}

Based on pro-viral actions playing by DPP4/CD26 during SARSCoV-2 infection, the perspective of blocking this glycoprotein might be clinically relevant. Theoretically, three different approaches could be utilized:

\section{i) DPP4 inhibitors.}

ii) Anti-CD26 monoclonal antibodies, such as Begelomab, and

iii) Adenosine deaminase agonists.

\section{DPP4 Inhibitors}

In the last months, many groups theorized the use of DPP4 inhibitors for COVID-19 pandemic, considering their antiinflammatory power previously demonstrated in diabetes mellitus [73]. DPP4 inhibitors reduce oxidative stress and inflammation, as demonstrated by reduction in diabetic patients of IL-6, IL-18, CRP, ICAM-1 and TNF alpha and IL10 increased levels [74,75]. Moreover, DPP4 inhibitors modulate production of collagen induced by TGF beta, and switch macrophages from M1 (pro-inflammatory) to M2 (anti- inflammatory) phenotype. In a murine model, all above activities lead to improvement of bleomycin-induced dermal and pulmonary fibrosis [76]. DPP4 inhibitors are classified in 3 different classes according to their tridimensional structures. In this respect, vildagliptin and saxagliptin belongs to class 1 , alogliptin and linagliptin to class 2 , while class 3 is represented by sitagliptin, gemigliptin, anagliptin, and teneligliptin. Teneligliptin has been reported to play an anti-atherosclerotic effect by increasing adiponectin and reducing selectins, VCAM-1 and PAI-1 [77], while vildagliptin can control transplanted lung rejection by lowering production of TNF alpha [78]. All these positive effects were not significantly affected by the onset of severe adverse events [79], except for a significant association with the bullous pemphigoid, reported for vildagliptin, in a lower measure for linagliptin, while it was not documented during sitagliptin treatment, thus resulting the best candidate for COVID-19 patients [80].

The role of anti-diabetic compounds in the 2020 pandemic has been recently extensively reviewed [81]. The authors promote metformin. However, some caution should be used in unstable cases and in patients with concomitant sepsis, due to its possible negative effect on the host immunocompetence, as showed by a reduced response to influenza vaccination observed in diabetic subjects receiving this drug [82]. Moreover, they suggested a possible rapid translation into the clinical practice of the Glucagon-like peptide 1 receptor (GLP-1R) agonists, such as liraglutide and exenatide, due to their powerful anti-inflammatory effect [83]. Indeed, in an Alzheimer animal model, liraglutide protected brain against amyloid accumulation [84]. The same drug reduced the intimal hyperplasia and levels of TNF alpha, IL6, IL1, and IL18 in a myocardial ischemia model [85]. Insulin is also suggested for the most severe COVID-19 cases, based on previous experiences demonstrating its positive impact in significantly reducing morbidity and mortality rate in critically ill patients [86].

\section{Anti-CD26 monoclonal antibodies}

Another conceivable way to counterbalance the negative effects of the activation of Coronavirus/DPP4/CD26 axis is represented by the receptor blockage through the anti- CD26 monoclonal antibodies. In T-cell lymphoma, the murine antibody IF7 was able to inhibit tumor cell growth and to prolong survival of tumorbearing mice [87]. In the pleural mesothelioma model, the antiCD26 antibody reduced neoplastic cell growth by enhancing cyclin-dependent kinase inhibitor p21 and downregulating the ubiquitin-specific protease 22 [88]. In multiple myeloma, the anti-CD26 antibody inhibited osteoclast differentiation, especially when used in combination with Dexamethasone, Bortezomib or Lenalidomide [89]. But the success of the anti-CD26 antibodies as anti-inflammatory compounds is particularly evident in the aGVHD, condition that mimics the cytokine storm detected in COVID-19 patients. In a murine model, the GVHD symptoms were associated with the increased CD26 expression on T lymphocytes and on GVHD target tissues. Administration of the anti-CD26 antibody decreased aGVHD severity and prolonged mice survival preserving the graftversus-leukemia effect [90]. Recently, a commercially available 
anti- CD26 monoclonal antibody, Begelomab, has been employed for treating steroid-refractory aGVHD patients. Among them, 28 cases were treated in two prospective trials and 41 were enrolled in a compassionate use study. aGVHD was of grade-II in 8, grade-III in 33 , and grade-IV in 28 patients.

In the compassionate use, Begelomab was administered at $3 \mathrm{mg} / \mathrm{m}^{2} /$ day for 5 days, followed by six additional doses of $3 \mathrm{mg} /$ $\mathrm{m}^{2}$ at day $+10,+14,+17,350+21$, and +24 . The overall response rate at one month was respectively $75 \%$ in the prospective studies and $61 \%$ in the compassionate use, with a similar complete response rates (11 and 12\%, respectively). Response in grade-III GVHD was higher than $70 \%$, and response in grade-IV GVHD cases about $60 \%$, with higher response rates described for skin, liver, and gut. The drug was well tolerated, with the most common adverse events being diarrhea, cytomegalovirus reactivation, infections, more likely associated to aGVHD [91]. Nevertheless, CD26 shedding could be an issue, since high levels of circulating CD26 was detected during Begelomab administration. Indeed, the shedding CD26 form could rapidly bind the monoclonal antibody, potentially reducing its therapeutic effect. Studies on anti-CD26 dose-adjusted based on soluble CD26 levels might be hypothesized for achieving higher rate of success.

\section{Adenosine Deaminase Agonists}

Adenosine deaminase (ADA), the enzyme that catalyzes the hydrolytic deamination of adenosine to inosine, represents an additional viable path to targeting DPP4/CD26 [92]. ADA is a substrate of the proteolytic activity of DPP4; when it is hyperactivated (as occurs during viral infection), ADA levels decrease, with the consequent increase of adenosine levels. Because high adenosine concentrations inhibit T-cells proliferation [93], we postulate that SARS-CoV-2 might use CD26 path for further inhibiting the host immune esponse. Thus, ADA agonists could hold additional therapeutic room for COVID-19. ADA,

a natural antagonist for DPP4, has been previously reported to reduce the respiratory syndrome sustained by the SARS-CoV [94]. In this respect, some suggestions might be derived from the treatment options of human severe combined immunodeficiency associated with mutations occurring in the ADA gene (ADASCID), and characterized by profound lymphopenia and immune dysregulation [95]. ADA-SCID therapeutic armamentarium includes enzyme replacement, allogeneic hematopoietic stem cell transplantation, and, more recently, gene therapy [96]. Although all the above-mentioned approaches are not applicable, the pegylated formulation of ADA (30 U/kg twice weekly), employed in the past with remarkable results and no adverse events [97] could represent a further reasonable approach to the COVID-19. All the above reported data support the hypothesis that SARS-CoV-2 makes a strict 'pact with the devil', namely with the glycoprotein DPP4/ CD26, which in turn represents the first door for its entry into the human 'friend/foe'. Once entered into the host cell, DPP4/CD26 helps the virus to downgrading autophagy, sustaining inflammation and fighting the host immune response (Figure 1A).

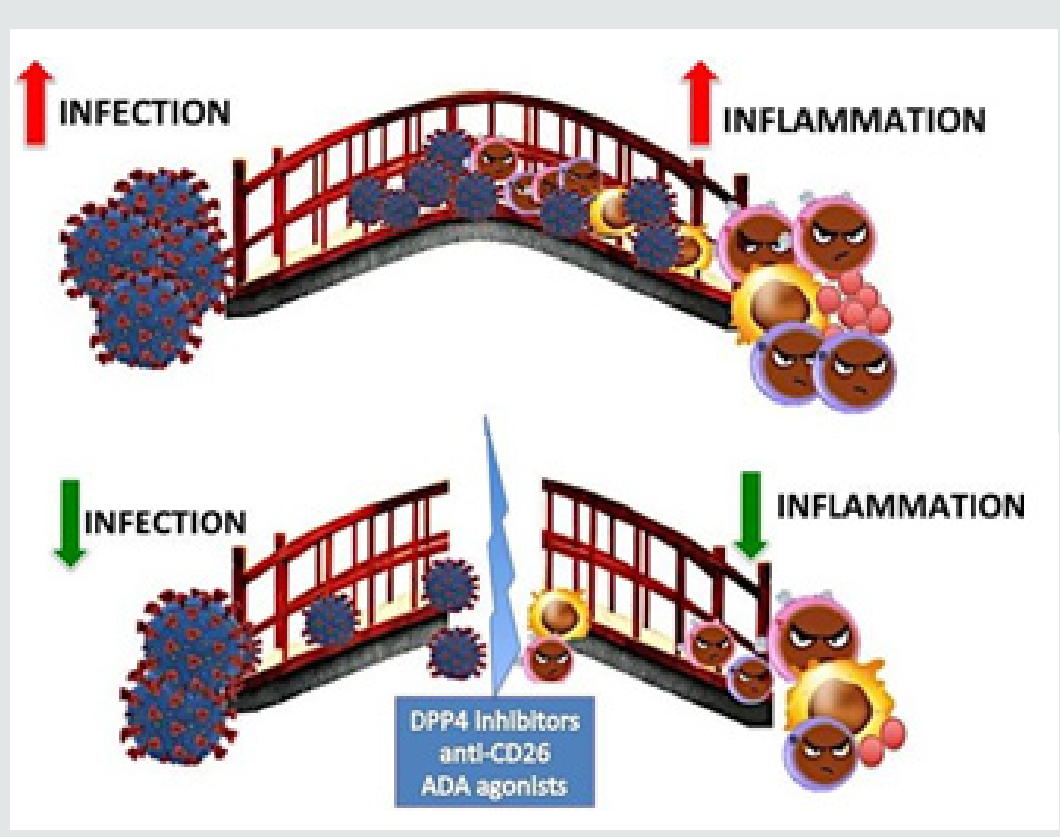

Figure 1: In the figure DPP4/CD26 is depicted as the bridge that connects Coronavirus 727 (on the left side) to the immune response/inflammation actors (B lymphocytes in violet, T 728 lymphocytes in pink, macrophages in yellow, cytokines in red). In the upper part of the 729 figure (A), when the bridge is intact, virus sustains inflammation and the excessive immune 730 response, with a great damage for the host (COVID-19). When the DPP4/CD26 bridge is 731 destroyed (by DPP4 inhibitors, anti-CD26, ADA agonists), the soldiers from both parts are 732 defeated, and an equilibrated immune response restored. The final balance is a powerful 733 benefic effect against COVID-19. 


\section{Conclusion}

In conclusion, different strategies are ideally available to potentially breaking down the SARS-COV-2/CD26 engagement (Figure 1B). Both bridge sides are theoretically weak enough to build up a key tactic to attack the virus-DPP4 axis. To date, we could imagine stopping the virus before crossing the bridge by social distancing interventions. Moreover, when the virus is approaching the bridge, vaccine-derived neutralizing antibodies, hopefully soon available, will provide the ultimate solution. However, by the host bridge side, we might imagine to counteract SARSCOV-2 attack also by anti-CD26 compounds, that might represent useful bullets to be fired from the first barricade, represented by autophagy and adequate immune response (Figure 1A). Although drug repurposing has emerged as a more convenient cost-effective as well as a faster drug discovery approach, clinical trials are warranted for demonstrating if theories can be translated into therapeutic options in daily practice or to remain interesting nevertheless merely scientific speculations.

\section{Acknowledgments}

This manuscript is dedicated to all people that everyday fight the war against Coronavirus and cystic fibrosis.

\section{Conflicts of Interest}

Authors do not have any conflict of interest to declare.

\section{Authors Contribution}

SG and FM wrote the manuscript; all authors collaborated to the discussion and revised the article.

\section{References}

1. De Wit E, Van Doremalen N, Falzarano D, Munster VJ (2016) SARS and MERS: recent insights into emerging coronaviruses. Nat Rev Microbiol 14:523-534.

2. Yuan OM, Wu NC, Zhu X, Lee CCD, So RTY, et al. (2020) A HighlyConserved Cryptic Epitope in the Receptor-Binding Domains of SARS-CoV-2 and SARS-CoV. Science pp: eabb7269.

3. Qi F, Qian S, Zhang S, Zhang Z (2020) Single Cell RNA Sequencing of 13 Human Tissues Identify Cell Types and Receptors of Human Coronaviruses. BiochemBiophysResCommun 526(1):135-140.

4. Berend JB, Smits SL, Haagmans BL (2014) Membrane ectopeptidases targeted by human coronaviruses. Curr Opin Virol 6:55-60.

5. Trzaskalski NA, Fadzeyeva E, Mulvihill EE (2020) Dipeptidyl Peptidase-4 at the Interface Between Inflammation and Metabolism. Clin Med Insights Endocrinol Diabetes 13:1179551420912972.

6. Ou X, Liu Y, Lei X, Li P, Mi D, et al. (2020) Characterization of spike glycoprotein of SARS-CoV-2 on virus entry and its immune crossreactivity with SARS-CoV. Nat Commun 11:1620.

7. Yang N, Shen MM (2020) Targeting the Endocytic Pathway and Autophagy Process as a N ovel Therapeutic Strategy in C OVID-19. Int J B iol S ci 16:1 724-1731.

8. Touret F, D e L amballerie X (2020) O f c hloroquine a nd C OVID-19. A ntiviral Res 177:104762.
9. Baron SA, Devaux, Colson P, Raoult D, Rolain JM (2020) Teicoplanin: an alternative drug for the treatment of coronavirus COVID-19? Int J Antimicrob Agents Mar 13: 105944.

10. Chen N, Zhou M, Dong X, Qu J, Gong F, et al. (2020) Epidemiological and clinical characteristics of 99 cases of 2019 novel coronavirus pneumonia in Wuhan, China: a descriptive study. Lancet 395:507-513.

11. Tang N, Bai H, Chen X, Gong J, Li D, et al. (2020) Anticoagulant treatment is associated with decreased mortality in severe coronavirus disease 2019 patients with coagulopathy. J Thromb Homeostasis 18(5):1094-1099.

12. Misra DP, Agarwal V, Gasparyan AY, Zimba O (2020) Rheumatologists' perspective on coronavirus disease 19 (COVID-19) and potential therapeutic targets. Clin Rheumatol 10:1-8.

13. Liu J, Li S, Liu J, Liang B, Wang X, et al. (2020) Longitudinal characteristics of lymphocyte responses and cytokine profiles in the peripheral blood of SARS-CoV-2 infected patients. EBioMedicine.

14. Meisel SR, Shapiro H, Radnay J, Neuman Y, Khaskia AR, et al. (1998) Increased Expression of Neutrophil and Monocyte Adhesion Molecules LFA- 1 and Mac- 1 and Their Ligand ICAM- 1 and VLA-4 Throughout the Acute Phase of Myocardial Infarction: Possible Implications for Leukocyte Aggregation and Microvascular Plugging. Journal of the American College of Cardiology 31(1):120-125.

15. Sarzi-Puttini P, Giorgi V, Sirotti S, Marotto D, Ardizzone S, et al. (2020) COVID-19, Cytokines and Immunosuppression: What Can We Learn from Severe Acute Respiratory Syndrome? Clin Exp Rheumatol 38:337342 .

16. Scott LJ (2017) Tocilizumab: A Review in Rheumatoid Arthritis. Drugs 77(7):1865-1879.

17. Sönmez HE, Demir S, Bilginer Y, Özen S (2018) Anakinra treatment in macrophage activation syndrome: a single center experience and systemic review of literature. Clin Rheumatol 37:3329-3335.

18. Blauvelt A, Papp KA, Griffiths CE, Randazzo B, Wasfi Y, et al. (2017) Efficacy and safety of guselkumab, an anti-interleukin-23 monoclonal antibody, compared with adalimumab for the continuous treatment of patients with moderate to severe psoriasis: Results from the phase III, double-blinded, placebo- and active comparator-controlled VOYAGE 1 trial. J Am Acad Dermatol 76(3):405-417.

19. Ajayi S, Becker H, Reinhardt H, Engelhardt M, Zeiser R, et al. (2018) Ruxolitinib. Recent Results Cancer Res 212:119-132.

20. Lin L, Lu L, Cao W, Li T (2020) Hypothesis for potential pathogenesis of SARS-CoV-2 infection-a review of immune changes in patients with viral pneumonia. Emerg Microbes Infect 9(1):727-732.

21. Von Bubnoff N, Ihorst G, Grishina O, Röthling N, Bertz H, et al. (2018) Ruxolitinib in GvHD (RIG) study: a multicenter, randomized phase 2 trial to determine the response rate of Ruxolitinib and best available treatment (BAT) versus BAT in steroid-refractory acute graft-versushost disease (aGvHD) (NCT02396628). BMC Cancer 18(1):1130-1132.

22. Qi SY, Riviere PJ, Trojnar J, Junien JL, Akinsanya KO (2003) Cloning and characterization of dipeptidyl peptidase 10, a new member of an emerging subgroup of serine proteases. Biochem J 373(pt1):179-189.

23. Zhao Y (2019) CD26 in autoimmune diseases: The other side of "moonlight protein". Int Immunopharmacol 75(1):105757.

24. Xia C, Goud A, D'Souza J, Dahagam C, Rao X, et al. (2017) DPP4 inhibitors and cardiovascular outcomes: safety on heart failure. Heart Fail Rev 22(3):299-304.

25. Silva Júnior WS, Godoy-Matos AF, Kraemer-Aguiar LG (2015) Dipeptidyl Peptidase 4: A New Link between Diabetes Mellitus and Atherosclerosis? Biomed Res Int 816164.

26. Aliyari SZ, Ebrahimi KA, Mehdipour A, Nozad CH (2017) Regulation and roles of CD26/DPPIV in hematopoiesis and diseases. Biomed Pharmacother 91:88-94. 
27. Deacon CF (2019) Physiology and Pharmacology of DPP-4 in Homeostasis and the Treatment of Type 2 Diabetes. Frontiers Endocrinol (Lausanne) 10:80.

28. Seys LJM, Widagdo W, Verhamme FM, Kleinjan A, Janssens W, et al. (2018) DPP4, the Middle East Respiratory Syndrome Coronavirus Receptor, is Upregulated in Lungs of Smokers and Chronic Obstructive Pulmonary Disease Patients. Clin Infect Dis 66:45-53.

29. Meyerholz DK, Lambertz AM, McCray PB (2016) Dipeptidyl Peptidase 4 Distribution in the Human Respiratory Tract: Implications for the Middle East Respiratory Syndrome. Am J Pathol 186(1):78-86.

30. Zou H, Zhu N, Li S (2020) The emerging role of dipeptidyl-peptidase-4 as a therapeutic target in lung disease. Expert Opin Ther Targets 24(2):147-153.

31. Proost P, De Meester I, Schols D, Struyf S, Lambeir AM, et al. (1998) Amino-terminal truncation of chemokines by CD26/dipeptidylpeptidase IV. Conversion of RANTES into a potent inhibitor of monocyte chemotaxis and HIV-1-infection. J Biol Chem 273(13):7222-7227.

32. Caì Y, Yú SQ Postnikova EN, Mazur S, Bernbaum JG, et al. (2014) CD26/ DPP4 cell-surface expression in bat cells correlates with bat cell susceptibility to Middle East respiratory syndrome coronavirus (MERS$\mathrm{CoV}$ ) infection and evolution of persistent infection. PLoS One 9(11) e112060.

33. Raj VS, Mou H, Smits SL, Dekkers DH, Müller MA, et al. (2013) Dipeptidyl peptidase 4 is a functional receptor for the emerging human coronavirusEMC. Nature 495(7440):251-254.

34. Vankadari N, Wilce JA (2020) Emerging WuHan (COVID-19) coronavirus: glycan shield and structure prediction of spike glycoprotein and its interaction with human CD26. Emerg Microbes Infect 9(1): 601-604.

35. Kim Y, Cheon S, Min CK, Sohn KM, Kang YJ, et al. (2016) Spread of Mutant Middle East Respiratory Syndrome Coronavirus with Reduced Affinity to Human CD26 during the South Korean Outbreak. mBio 7(2): e00019.

36. Ou X, Liu Y, Lei X, Li P, Mi D, et al. (2020) Characterization of spike glycoprotein of SARS-CoV-2 on virus entry and its immune crossreactivity with SARS-CoV. Nat Commun 11(1): 1600-1620.

37. Chiu RW, Tang NL, Hui DS, Chung GT, Chim SS, et al. (2004) ACE2 gene polymorphisms do not affect outcome of severe acute respiratory syndrome. Clin Chem 50(9):1683-1686.

38. Ahmed RH, Huri HZ, Al Hamodi Z, Salem SD, Al-Absi B, et al. (2016) Association of DPP4 Gene Polymorphisms with Type 2 Diabetes Mellitus in Malaysian Subjects. PLoS One 11(4): e0154369.

39. Nakamura A, Osonoi T, Terauchi Y (2010) Relationship between urinary sodium excretion and pioglitazone-induced edema. J Diabetes Investig 1(5): 208-211.

40. Vijayan D, Young A, Teng MWL, Smyth MJ (2017) Targeting immunosuppressive adenosine in cancer. Nat Rev Cancer 17(12): 709724.

41. Nistala R, Savin V (2017) Diabetes, hypertension, and chronic kidney disease progression: role of DPP4. Am J Physiol Renal Physiol 312(4): F661-F670.

42. Aliyari Serej Z, Ebrahimi Kalan A, Mehdipour A, Nozad Charoudeh H (2017) Regulation and roles of CD26/DPPIV in hematopoiesis and diseases. Biomed Pharmacother 91: 88-94.

43. Neuhaus M, Munder A, Schipke J, Schmiedl A (2019) Lung infection caused by Pseudomonas aeruginosa in a CD26/DPP4 deficient F344 rat model. Inflamm Re 68(7): 529-544.

44. Pascual-Ramos V, Hernández-Cruz B, Villalobos I, Sifuentes-Osornio J, Alcocer-Varela J (2002) Purified protein derivative reaction in systemic lupus erythematosus patients. Indirect study of cellular immunity. Lupus 11(1):25-30.
45. Zhao Y (2019) D26 in autoimmune diseases: The other side of "moonlight protein". Int Immunopharmacol 75:105757.

46. Gerli R, Muscat C, Bertotto A, Bistoni O, Agea E, et al. (1996) CD26 surface molecule involvement in $\mathrm{T}$ cell activation and lymphokine synthesis in rheumatoid and other inflammatory synovitis. Clin Immunol Immunopathol 80(1):31-37.

47. Nieto-Fontarigo JJ, González-Barcala FJ, San José E, Arias P, Nogueira M, et al. (2019) CD26 and Asthma: a Comprehensive Review. Clin Rev Allergy Immuno 56(2):139-160.

48. Nielsen BR, Ratzer R, Börnsen L, Von Essen MR, Christensen JR, Sellebjerg F (2017) Characterization of naïve, memory and effector T cells in progressive multiple sclerosis. J Neuroimmunol 310:17-25.

49. Jin Y, Zhao H, Hou Y, Song G (2020) The effects of dipeptidyl peptidase4inhibitors and glucagon-like peptide 1 receptor agonists on cognitive functions in adults with type 2 diabetes mellitus: a systematic review and meta-analysis. Acta Diabetol.

50. Nistala R, Savin V (2017) Diabetes, hypertension, and chronic kidney disease progression: role of DPP4. Am J Physiol Renal Physiol 312(4): F661-F670.

51. Roncon L, Zuin M, Rigatelli G, Zuliani G (2020) Diabetic patients with COVID-19 infection are at higher risk of ICU admission and poor shortterm outcome. J Clin Virol 127:104354.

52. Murata M (2018) Inflammation and cancer. Environ Health Prev Med 23:50.

53. Wilson MJ, Ruhland AR, Quast BJ, Reddy PK, Ewing SL, et al. (2000) Dipeptidylpeptidase IV activities are elevated in prostate cancers and adjacent benign hyperplastic glands. J Androl 21(2): 220-226.

54. Aratake Y, Kotani T, Tamura K, Araki Y, Kuribayashi T, et al. (1991) Dipeptidyl aminopeptidase IV staining of cytologic preparations to distinguish benign from malignant thyroid diseases. Am J Clin Pathol 96(3):306-310.

55. Kondo S, Kotani T, Tamura K, Aratake Y, Uno H, et al. (1996) Expression of CD26/dipeptidyl peptidase IV in adult T cell leukemia/lymphoma (ATLL). Leuk Res 20(4): 357-363.

56. Cedeno-Laurent F, Wysocka M, Obstfeld AE, Novoa RA, Vittorio CC, et al. (2017) Gain of CD26 expression on the malignant T-cells in relapsed erythrodermic leukemic mycosis fungoides. J Cutan Pathol 44(5):462466.

57. Herrmann H, Sadovnik I, Cerny-Reiterer S, Rülicke T, Stefanzl G, et al. (2014) Dipeptidylpeptidase IV (CD26) defines leukemic stem cells (LSC) in chronic myeloid leukemia. Blood 123(25): 3951-3962.

58. Galimberti S, Grassi S, Baratè C, Guerrini F, Ciabatti E, et al. (2018) The Polycomb BMI1 Protein Is Co-expressed With CD26+ in Leukemic Stem Cells of Chronic Myeloid Leukemia. Front Oncol 8:550-555.

59. Galimberti S, Grassi S, Baratè C, Guerrini F, Ciabatti E, et al. (2018) The Polycomb BMI1 Protein Is Co-expressed With CD26+ in Leukemic Stem Cells of Chronic Myeloid Leukemia. Front Oncol 8:550-555.

60. Bocchia M, Sicuranza A, Abruzzese E, Iurlo A, Sirianni S, et al. (2018) Residual Peripheral Blood CD26+ Leukemic Stem Cells in Chronic Myeloid Leukemia Patients During TKI Therapy and During TreatmentFree Remission. Front Oncol 8:194.

61. Valent P, Sadovnik I, Ráčil Z, Herrmann H, Blatt K, et al. (2014) DPPIV (CD26) as a novel stem cell marker in $\mathrm{Ph}+$ chronic myeloid leukaemia. Eur J Clin Invest 44(12): 1239-1245.

62. Willmann M, Sadovnik I, Eisenwort G, Entner M, Bernthaler T, et al. (2018) Evaluation of cooperative antileukemic effects of nilotinib and vildagliptin in Ph+ chronic myeloid leukemia. Exp Hematol 57:50-59. 
63. Matuszak M, Lewandowski K, Czyż A, Kiernicka-Parulska J, PrzybyłowiczChalecka A, et al. (2016) The prognostic significance of surface dipeptidylpeptidase IV (CD26) expression in B-cell chronic lymphocytic leukemia. Leuk Res 47:166-171.

64. Cro L, Morabito F, Zucal N, Fabris S, Lionetti M, et al. (2009) CD26 expression in mature B-cell neoplasia: its possible role as a new prognostic marker in B-CLL. Hematol Oncol 27(3):140-147.

65. Gassen NC, Papies J, Bajaj T, Dethloff F, Jackson E, et al. (2020) Analysis of SARS-CoV-2-controlled autophagy reveals spermidine, MK-2206, and niclosamide as putative antiviral therapeutics. BioRxiv.

66. Ge Y, Huang M, Yao YM (2018) Autophagy and proinflammatory cytokines: Interactions and clinical implications. Cytokine Growth Factor Rev 43:38-46.

67. Qian M, Fang X, Wang X (2017) Autophagy and inflammation. Clin Transl Med 6:24.

68. De Haan CA, Reggiori F (2008) Are nidoviruses hijacking the autophagy machinery? Autophagy 4(3):276-279.

69. Shi CS, Qi HY, Boularan C, Huang NN, Abu Asab M, et al. (2014) SARScoronavirus open reading frame-9b suppresses innate immunity by targeting mitochondria and the MAVS/TRAF3/TRAF6 signalosome. J Immunol 193(6):3080-3089.

70. Gassen NC, Niemeyer D, Muth D, Corman VM, Martinelli S, et al. (2019) SKP2 attenuates autophagy through Beclin1-ubiquitination and its inhibition reduces MERS-Coronavirus infection. Nat Commun 10(1):5756-5770.

71. Pietrocola F, Lachkar S, Enot DP, Niso Santano M, Bravo-San Pedro JM, et al. (2015) Spermidine induces autophagy by inhibiting the acetyltransferase EP300. Cell Death Differ 22(3):509-516.

72. Murase H, Kuno A, Miki T, Tanno M, Yano T, et al. (2015) Inhibition of DPP-4 reduces acute mortality after myocardial infarction with restoration of autophagic response in type 2 diabetic rats. Cardiovasc Diabeto 14: 99-103.

73. Dai X, Zeng J, Yan X, Lin Q, Wang K, et al. (2018) Sitagliptin-mediated preservation of endothelial progenitor cell function via augmenting autophagy enhances ischaemic angiogenesis in diabetes. J Cell Mol Med 22(1):89-100.

74. Tomovic K, Lazarevic J, Kocic G, Deljanin-Ilic M, Anderluh M, et al. (2019) Mechanisms and pathways of anti-inflammatory activity of DPP-4 inhibitors in cardiovascular and renal protection. Med Res Rev 39(1): 404-422.

75. Tremblay AJ, Lamarche B, Deacon CF, Weisnagel SJ, Couture P (2014) Effects of sitagliptin therapy on markers of low-grade inflammation and cell adhesion molecules in patients with type 2 diabetes. Metabolism 63(9): 1141-1148

76. Satoh Asahara N, Sasaki Y, Wada H, Tochiya M, Iguchi A, et al. (2013) A dipeptidyl peptidase-4 inhibitor, sitagliptin, exerts anti-inflammatory effects in type 2 diabetic patients. Metabolism 62(3):347-351.

77. McHugh J (2019) DDP4 inhibition reduces fibrosis. Nat Rev Rheumatol 15(10): 570-575.

78. Salim HM, Fukuda D, Higashikuni Y, Tanaka K, Hirata Y, et al. (2017) Teneligliptin, a dipeptidyl peptidase-4 inhibitor, attenuated proinflammatory phenotype of perivascular adipose tissue and inhibited atherogenesis in normoglycemic apolipoprotein-E-deficient mice. Vascul Pharmacol 96-98: 19-25.

79. Yamada Y, Jang JH, De Meester I, Baerts L, Vliegen G, et al. (2016) CD26 costimulatory blockade improves lung allograft rejection and is associated with enhanced interleukin-10 expression. J Heart Lung Transplant 35(4): 508-17.

80. Hayashi T, Murayama H, Shinfuku Y, Taniguchi T, Tsumiyama I, et al (2020) Safety and efficacy of vildagliptin: 52-week post-marketing surveillance of Japanese patients with type 2 diabetes in combination with other oral antidiabetics and insulin. Expert Opin Pharmacother 21(1): 121-130.

81. Phan K, Charlton O, Smith SD. (2020) Dipeptidyl peptidase-4 inhibitors and bullous emphigoid: A systematic review and adjusted meta-analysis. Australasian Journal of Dermatology 61(1): e15-e21.

82. Drucker DJ (2020) Coronavirus infections and type 2 diabetes-shared pathways with therapeutic implications. Endocr Rev 41(3).

83. Chen P, Lin L, Xu X, Zhang Z, Cai W, et al. (2020) Liraglutide improved inflammation via mediating IL-23/Th-17 pathway in obese diabetic mice with psoriasiform skin. J Dermatolog Treat p:1-7.

84. Duarte AI, Candeias E, Alves IN, Mena D, Silva DF, et al (2020) Liraglutide Protects Against Brain Amyloid- $\beta 1-42$ Accumulation in Female Mice with Early Alzheimer's Disease-Like Pathology by Partially Rescuing Oxidative/Nitrosative Stress and Inflammation. Int J Mol Sci 21(5).

85. Xia J, Li Q, Liu Y, Ren Q, Gao J, et al (2020) A GLP-1 Analog Liraglutide Reduces Intimal Hyperplasia After Coronary Stent Implantation via Regulation of Glycemic Variability and NLRP3 Inflammasome/IL-10 Signaling in Diabetic Swine. Front Pharmacol 11: 360-372.

86. Hansen TK, Thiel S, Wouters PJ, Christiansen JS, Van den Berghe G (2003) Intensive insulin therapy exerts anti-inflammatory effects in critically ill patients and counteracts the adverse effect of low mannose-binding lectin levels. J Clin Endocrinol Metab 88(3):1082-1088.

87. Ho L, Aytac U, Stephens LC, Ohnuma K, Mills GB, et al. (2001) In vitro and in vivo antitumor effect of the anti-CD26 monoclonal antibody 1F7 on human CD30+ anaplastic large cell T-cell lymphoma Karpas 299. Clin Cancer Res 7(7):2031-2040.

88. Okamoto T, Yamazaki H, Hatano R, Yamada T, Kaneko Y, et al. (2018) Targeting CD26 suppresses proliferation of malignant mesothelioma cell via downmodulation of ubiquitin-specific protease 22. Biochem Biophys Res Commun 504(2):491-498.

89. Ann EJ, Kim HY, Choi YH, Kim MY (2011) Inhibition of Notch1 signaling by Runx2 during osteoblast differentiation. J Bone Miner Res 26:317330 .

90. Hatano R, Ohnuma K, Yamamoto J, Dang NH, Yamada T, et al. (2013) Prevention of acute graft-versus-host disease by humanized anti-CD26 monoclonal antibody. British Journal of Haematology 162(2):263-277.

91. Bacigalupo A, Angelucci E, Raiola AM, Varaldo R, Di Grazia C, et al. (2020) Treatment of steroid resistant acute graft versus host disease with an anti-CD26 monoclonal antibody-Begelomab. Bone Marrow Transplant.

92. Cristalli G, Costanzi S, Lambertucci C, Lupidi G, Vittori S, et al. (2001) Adenosine deaminase: functional implications and different classes of inhibitors. Med Res Rev 21(2):105-128

93. Shao S, Xu Q, Yu X, Pan R, Chen Y (2020) Dipeptidyl peptidase 4 inhibitors and their potential immune modulatory functions. Pharmacolology \& Therapeutics 209: 107503

94. Raj VS, Smits SL, Provacia LB, Van Den Brand JM, Wiersma L, et al. (2014) Adenosine deaminase acts as a natural antagonist for dipeptidyl peptidase 4-mediated entry of the Middle East respiratory syndrome coronavirus. J Virol 88(3):1834-1838.

95. Flinn AM, Gennery AR (2018) Adenosine deaminase deficiency: a review. Orphanet J Rare Dis 13(1):60-65.

96. Bradford KL, Moretti FA, Carbonaro Sarracino DA, Gaspar HB, Kohn DB (2017) Adenosine Deaminase (ADA)-Deficient Severe Combined Immune Deficiency (SCID): Molecular Pathogenesis and Clinical Manifestations. J Clin Immunol 37(7):626-637.

97. Hershfield MS (1995) PEG-ADA replacement therapy for adenosine deaminase deficiency: an update after 8.5 years. Clin Immunol Immunopathol 76(3 Pt 2): S228-S232. 
(c) (P) This work is licensed under Creative Commons Attribution 4.0 License

To Submit Your Article Click Here:

Submit Article

DOI: $10.32474 /$ LOJPCR.2020.02.000128

Lupine Online Journal of
Pharmacology \& Clinical Research
Assets of Publishing with us
- Global archiving of articles
- Immediate, unrestricted online access
- Rigorous Peer Review Process
Pharmacology \& Clinical Research

